\title{
5D Parameter Estimation of Near-Field Sources Using Hybrid Evolutionary Computational Techniques
}

\author{
Fawad Zaman $^{1}$ and Ijaz Mansoor Qureshi ${ }^{2}$ \\ ${ }^{1}$ Department of Electronic Engineering, IIU, H-10, Islamabad 44000, Pakistan \\ ${ }^{2}$ Electrical Department, Air University, Islamabad 44000, Pakistan \\ Correspondence should be addressed to Fawad Zaman; fawad.phdee31@iiu.edu.pk
}

Received 30 August 2013; Accepted 1 December 2013; Published 18 February 2014

Academic Editors: M. F. G. Penedo and J. Tang

Copyright ( 2014 F. Zaman and I. M. Qureshi. This is an open access article distributed under the Creative Commons Attribution License, which permits unrestricted use, distribution, and reproduction in any medium, provided the original work is properly cited.

Hybrid evolutionary computational technique is developed to jointly estimate the amplitude, frequency, range, and 2D direction of arrival (elevation and azimuth angles) of near-field sources impinging on centrosymmetric cross array. Specifically, genetic algorithm is used as a global optimizer, whereas pattern search and interior point algorithms are employed as rapid local search optimizers. For this, a new multiobjective fitness function is constructed, which is the combination of mean square error and correlation between the normalized desired and estimated vectors. The performance of the proposed hybrid scheme is compared not only with the individual responses of genetic algorithm, interior point algorithm, and pattern search, but also with the existing traditional techniques. The proposed schemes produced fairly good results in terms of estimation accuracy, convergence rate, and robustness against noise. A large number of Monte-Carlo simulations are carried out to test out the validity and reliability of each scheme.

\section{Introduction}

Parameter estimation of signals is one of the key issues in array signal processing, which has direct applications in radar, sonar, seismic exploration, electronic surveillance, and so forth [1]. In the literature, various algorithms are available to discuss this issue, such as the MUSIC algorithm [2], the maximum likelihood (ML) algorithm [3], the matrix pencil (MP) algorithm [4], and the ESPRIT algorithm [5]. Many of these algorithms make a supposition that the sources are positioned in the far field of sensors array so that the signal received from them can be taken as plane waves. With this supposition, the wave front of each signal is only a function of the DOA of the sources, which is easy to deal with. However, the situation becomes complicated if the sources are situated closer to the sensor array (near field). In this case, the waves are considered to be spherical, where the wave-front of each signal is the function of DOA, as well as, range of the sources [6].

Many classical algorithms are also available to discuss the problem of near-field source localization, such as the linear prediction algorithm [7], the 2D MUSIC algorithm [8], and the ESPRIT based algorithms $[9,10]$. However, these algorithms mainly focus on 2D case, that is, estimation of the elevation angle and range parameters. Some algorithms are also available which deal with the 3D case (elevation angle, azimuth angle, and range) of near-field sources, for example, [11-14]. In [11] expectation-maximization (EM) algorithm is proposed, but it suffers from heavy computations and iterative process. A unitary ESPRIT algorithm is developed in [12] which requires further parameter pairing process, while the algorithm presented in [13] heavily relies on different carrier frequencies and approximated sinusoidal signals and also requires high sampling narrow band data. A spectral search based method is presented in [14] which can only be used for underwater environment. In [15], comparatively an efficient algorithm based on cumulants is proposed for 4D parameter estimation of near-field sources (frequency, range, and 2D DOA), but it also requires a large number of snapshots and ends up with higher mean square error (MSE). Moreover, it is also unable to estimate the amplitude of signals. 
Now to estimate the parameters of near-field sources, heuristic techniques like evolutionary computing techniques (ECT) can also be used in the field of optimization. ECT, which is also known as computational intelligence, is a subfield of artificial intelligence that can be employed for combinatorial as well as for continuous optimization problems. ECT has stochastic or metaheuristic optimization nature and is considered to be global optimization methods. These techniques include genetic algorithm (GA) [16], particle swarm optimization (PSO) [17], and differential evolution (DE) [18]. These techniques are based on the principle of biological evolution, such as genetic inheritance and natural selection. One of the most important features of ECT is that they become even more reliable and effective when hybridized with any other efficient scheme such as pattern search (PS), active set (AS), and interior point algorithm (IPA) [19-24].

In this paper, 5D parameters (amplitude, frequency, range, elevation angle, and azimuth angle) of near-field sources impinging on centrosymmetric cross array are jointly estimated. Initially we used GA, PS, and IPA alone, but then we adopt hybrid evolutionary computing techniques based on GA hybridized with PS or IPA. In these hybrid approaches, the solution starts with a global optimizer (GA) and ends up with local optimizers (PS or IPA). For this a new multiobjective fitness function is used, which is the combination of MSE and correlation between normalized desired and estimated vectors. It requires only a single snapshot, which obviously decreases the computational cost. The performances of these two hybrid approaches (GA-PS and GA-IPA) are compared not only with each other, but also with the individual performance of GA, IPA, and PS. Besides, the proposed hybrid schemes are also compared with the traditional techniques available in the literature [15].

Throughout the paper, matrices and vectors are represented by bold upper and lower case letters, respectively, whereas $T, H$, and $N$ are used, respectively, for transpose, hermitian, and normalization of vectors or matrix.

The rest of the work is organized as follows. In Section 2, data model is developed for near-field sources, while Section 3 describes the signal subspace dimension. The proposed schemes are given in Section 4, while results and simulations are provided in Section 5. Finally, conclusion and future work direction are given in Section 6.

\section{Signal Model for Near-Field Sources}

In this section, signal model for near-field sources impinging on centrosymmetric cross array (CSCA) is developed. All sources are considered to be narrow band and mutually statistically independent. The amplitude $(a)$, frequency $(f)$, range $(r)$, and $2 \mathrm{D} \operatorname{DOA}(\theta, \phi)$ are different for different sources. The CSCA is composed of two subarrays that are placed along $x$-axis and $y$-axis, respectively, as shown in Figure 1. The total number of sensors in the array is $4 Q+$ 1 where each subarray consists of $2 Q$ sensors, while the reference sensor is common among both. If $P$ is the total number of sources, then the signal received at $m$ th and $n$th

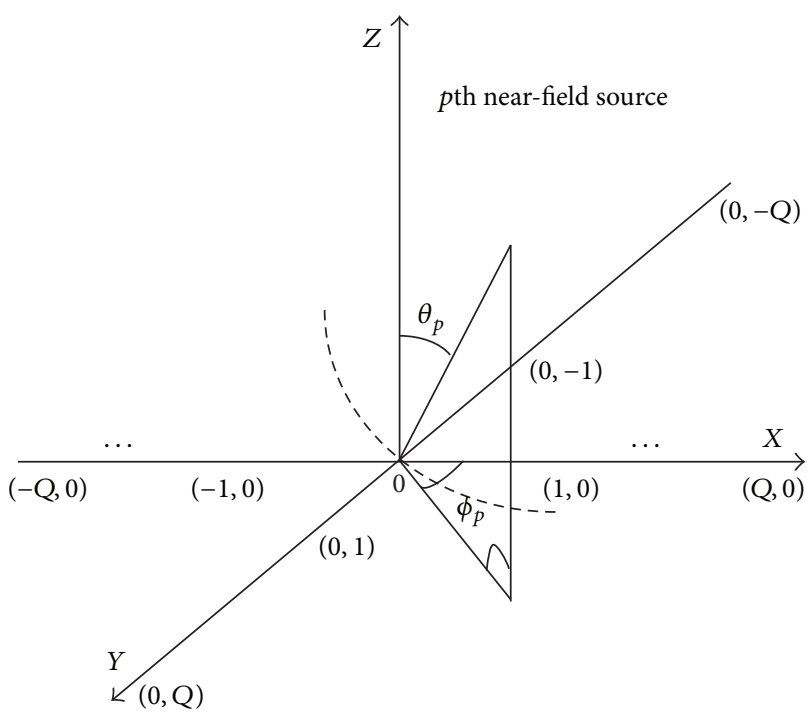

FIGURE 1: Signal model for near-field sources.

sensor in $x$-axis and $y$-axis subarrays, respectively, can be modeled as

$$
\begin{aligned}
w_{m, 0} & =\sum_{p=1}^{P} a_{p} e^{j\left(m \alpha_{x p}+m^{2} \beta_{x p}\right)}+\eta_{m, 0}, \\
w_{0, n} & =\sum_{p=1}^{P} a_{p} e^{j\left(n \alpha_{y p}+n^{2} \beta_{y p}\right)}+\eta_{0, n},
\end{aligned}
$$

where $\eta_{m, 0}$ and $\eta_{0, n}$ represent the additive white Gaussian noise (AWGN) added at $m$ th and $n$th sensors in $x$-axis and $y$-axis subarrays, respectively.

In (1), $\alpha_{x p}$ and $\beta_{x p}$ can be given as

$$
\alpha_{x p}=-k_{p} d \sin \theta_{p} \cos \phi_{p}
$$

In (3), $k_{p}=2 \pi / \lambda_{p}$, where $\lambda_{p}=c / f_{p}$. Similarly, $d=\lambda_{\min } / 4$, where $\lambda_{\min }=c / f_{\max }$. So, (3) can be represented as

$$
\alpha_{x p}=-\frac{\pi f_{p}}{2 f_{\max }}\left(\sin \theta_{p} \cos \phi_{p}\right) .
$$

In the same way,

$$
\beta_{x p}=\frac{\pi d^{2}\left(1-\sin ^{2} \theta_{p} \cos ^{2} \phi_{p}\right)}{\lambda_{p} r_{p}},
$$

where (5) can be further rewritten as

$$
\beta_{x p}=\frac{\pi f_{p}^{2}}{16 f_{\max } r_{p}}\left(1-\sin ^{2} \theta_{p} \cos ^{2} \phi_{p}\right) .
$$

Similarly, in (2), $\alpha_{y p}$ and $\beta_{y p}$ can be given as

$$
\begin{gathered}
\alpha_{y p}=-\frac{\pi f_{p}}{2 f_{\max }}\left(\sin \theta_{p} \sin \phi_{p}\right), \\
\beta_{y p}=\frac{\pi f_{p}^{2}}{16 f_{\text {max }} r_{p}}\left(1-\sin ^{2} \theta_{p} \sin ^{2} \phi_{p}\right) .
\end{gathered}
$$


By using (4) and ((6)-(7)) in (1) and (2), we get:

$$
\begin{aligned}
& w_{m, 0}=\sum_{p=1}^{P} a_{p} e^{j\left(\left(\pi / f_{\max }\right)\left(-\left(m f_{p} / 2\right)\left(\sin \theta_{p} \cos \phi_{p}\right)+\left(m^{2} f_{p}^{2} / 16 r_{p}\right)\left(1-\sin ^{2} \theta_{p} \cos ^{2} \phi_{p}\right)\right)\right)}+\eta_{m, 0} \\
& w_{0, n}=\sum_{p=1}^{P} a_{p} e^{j\left(\left(\pi / f_{\max }\right)\left(-\left(n f_{p} / 2\right)\left(\sin \theta_{p} \sin \phi_{p}\right)+\left(n^{2} f_{p}^{2} / 16 r_{p}\right)\left(1-\sin ^{2} \theta_{p} \sin ^{2} \phi_{p}\right)\right)\right)}+\eta_{0, n}
\end{aligned}
$$

where $f_{p}$ represents the frequency of pth source, while $f_{\max }$ is the maximum frequency to be used. In vector form (8) can be collectively represented as

$$
\mathbf{w}=\mathbf{B a}+\boldsymbol{\eta},
$$

where $\mathbf{w}, \boldsymbol{\eta}, \mathbf{a}$, and $\mathbf{B}$ can be defined as

$$
\begin{aligned}
& \mathbf{w}=\left[\begin{array}{lllll}
w_{-Q, 0} & w_{-Q+1,0} & \cdots & w_{-1,0} & w_{1,0}
\end{array}\right. \\
& \begin{array}{lllll}
w_{2,0} & \cdots & w_{\mathrm{Q}-1,0} & w_{\mathrm{Q}, 0} & w_{0,0}
\end{array} \\
& \begin{array}{llll}
w_{0,-Q} & w_{0,-Q+1} & \cdots & w_{0,-1}
\end{array} \\
& \left.\begin{array}{lllll}
w_{0,1} & w_{0,2} & \cdots & w_{0, \mathrm{Q}-1} & w_{0, \mathrm{Q}}
\end{array}\right]^{T}, \\
& \boldsymbol{\eta}=\left[\begin{array}{lllll}
\eta_{-\mathrm{Q}, 0} & \eta_{-\mathrm{Q}+1,0} & \cdots & \eta_{-1,0} & \eta_{1,0}
\end{array}\right. \\
& \begin{array}{lllll}
\eta_{2,0} & \cdots & \eta_{\mathrm{Q}-1,0} & \eta_{\mathrm{Q}, 0} & \eta_{0,0}
\end{array} \\
& \begin{array}{lllll}
\eta_{0,-\mathrm{Q}} & \eta_{0,-\mathrm{Q}+1} & \cdots & \eta_{0,-1} & \eta_{0,1}
\end{array} \\
& \left.\begin{array}{llll}
\eta_{0,2} & \cdots & \eta_{0, \mathrm{Q}-1} & \eta_{0, \mathrm{Q}}
\end{array}\right]^{T} \text {, } \\
& \mathbf{a}=\left[\begin{array}{llll}
a_{1} & a_{2} & \cdots & a_{P}
\end{array}\right]^{T}, \\
& \mathbf{B}=\left[\mathbf{b}\left(\alpha_{x 1}, \beta_{x 1}, \alpha_{y_{1}}, \beta_{y 1}\right), \ldots, \mathbf{b}\left(\alpha_{x p}, \beta_{x p}, \alpha_{y p}, \beta_{y p}\right)\right. \text {, } \\
& \left.\ldots, \mathbf{b}\left(\alpha_{x P}, \beta_{x P}, \alpha_{y_{P}}, \beta_{y P}\right)\right],
\end{aligned}
$$

where

$$
\begin{gathered}
\mathbf{b}\left(\alpha_{x p}, \beta_{x p}, \alpha_{y p}, \beta_{y p}\right) \\
=\left[e^{j\left[(-Q) \alpha_{x p}+(-Q)^{2} \beta_{x p}\right]}, e^{j\left[(-Q+1) \alpha_{x p}+(-Q+1)^{2} \beta_{x p}\right]},\right. \\
\ldots, e^{j\left[-\alpha_{x p}+\beta_{x p}\right]}, e^{j\left[\alpha_{x p}+\beta_{x p}\right]}, \ldots, \\
e^{j\left[(Q-1) \alpha_{x p}+(Q-1)^{2} \beta_{x p}\right]}, e^{j\left[(Q) \alpha_{x p}+(Q)^{2} \beta_{x p}\right]}, 1, \\
e^{j\left[(-Q) \alpha_{y p}+(-Q)^{2} \beta_{y p}\right]}, e^{j\left[(-Q+1) \alpha_{y p}+(-Q+1)^{2} \beta_{y p}\right]}, \\
\ldots, e^{j\left[-\alpha_{y p}+\beta_{y p}\right]}, e^{j\left[\alpha_{y p}+\beta_{y p}\right]}, \ldots, \\
\left.e^{j\left[(Q-1) \alpha_{y p}+(\mathrm{Q}-1)^{2} \beta_{y p}\right]}, e^{j\left[(Q) \alpha_{y p}+(Q)^{2} \beta_{y p}\right]}\right]^{T} .
\end{gathered}
$$

From (8), one can see that the unknown parameters are $a_{p}, f_{p}, r_{p}, \theta_{p}$, and $\phi_{p}$ where $p=1,2, \ldots, P$. So, the problem in hand is to estimate these 5D parameters jointly and efficiently; before starting the problem, it is important to find out the dimension of the signal subspace from the received snapshots.

\section{Signal Subspace Dimension}

For this purpose, we used nonparametric technique:

$$
\mathbf{w}=\mathbf{B a}+\boldsymbol{\eta},
$$

where a is a $P \times 1$ source vector, $\mathbf{B}$ is our $(4 Q+1) \times P$ array manifold matrix, and $\boldsymbol{\eta}$ is an AWGN vector with spectral matrix $\sigma_{\eta}^{2} I$. The spectral matrix of $\mathbf{w}$ is given as

$$
\mathbf{S}_{\mathbf{w}}(\omega)=\mathbf{B} \mathbf{A}(\omega) \mathbf{B}^{H}+\sigma^{2} \mathbf{I}=\mathbf{S}_{s}(\omega)+\mathbf{S}_{\eta}(\omega),
$$

where

$$
\mathbf{A}(\omega)=E\left[\mathbf{a}(\omega) \mathbf{a}^{H}(\omega)\right]
$$

We expect that the signals are incoherent, so that the rank of $\mathbf{S}_{s}(\omega)$ is equal to the number of signals. Let the rank of $\mathbf{S}_{s}(\omega)$ be $P$; then eigendecomposition of $\mathbf{S}_{\mathbf{w}}(\omega)$ is given as

$$
\mathbf{S}_{\mathbf{w}}(\omega)=\mathbf{Q}_{s} \Lambda_{s} \mathbf{Q}_{s}^{H}+\mathbf{Q}_{\eta} \Lambda_{\eta} \mathbf{Q}_{\eta}^{H},
$$

where

$$
\begin{gathered}
\boldsymbol{\Lambda}_{s}=\operatorname{diag}\left[\begin{array}{llll}
\sigma_{s_{1}}^{2} & \sigma_{s_{2}}^{2} & \cdots & \sigma_{s_{P}}^{2}
\end{array}\right] \\
\boldsymbol{\Lambda}_{\eta}=\operatorname{diag}\left[\begin{array}{llll}
\sigma_{\eta}^{2} & \sigma_{\eta}^{2} & \cdots & \sigma_{\eta}^{2}
\end{array}\right] \quad \text { with }(4 Q+1)-P \text { elements }
\end{gathered}
$$

$\mathbf{Q}_{s}$ has column vectors which are eigenvectors of $\mathbf{S}_{s}(\omega)$, and $\mathbf{Q}_{\eta}$ has column vectors which are eigenvectors of $\mathbf{S}_{n}(\omega)$. We expect the last $(4 Q+1)-P$ eigenvalues representing noise to be the smallest and also equal. For finding the dimensions of two subspaces, we can use the following hypothesis [25]:

$$
\begin{aligned}
L_{P}= & ((4 Q+1)-P) \ln \\
& \times\left[\frac{\left\{(1 /((4 Q+1)-P)) \sum_{l=P+1}^{4 Q+1} \lambda_{l}\right\}}{\left(\prod_{l=P+1}^{4 Q+1} \lambda_{l}\right)^{1 /((4 Q+1)-P)}}\right] .
\end{aligned}
$$

This numerator is the arithmetic mean of $(4 Q+1)-P$ being the smallest eigenvalues, while denominator is their geometric 


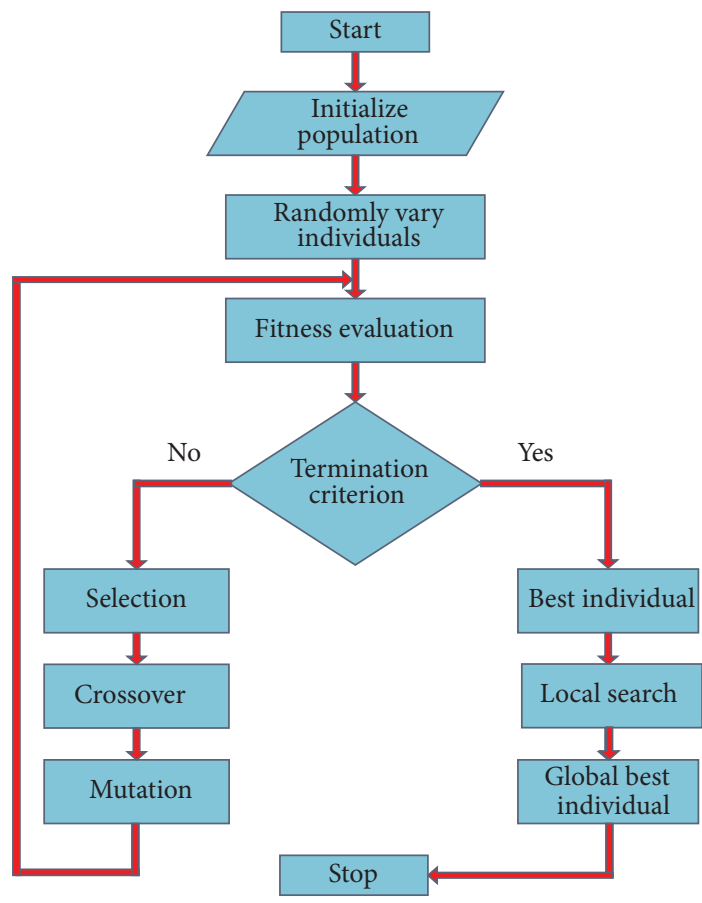

FIGURE 2: Flow diagram for hybrid GA-PS and GA-IPA.

mean. We start with $P=1$, then $P$ is chosen correctly, and then the last $(4 Q+1)-P$ eigen values are the smallest and equal, making $L_{P}(P)=0$. After having found $P$ by this test, we know exactly the number of signals; whether any of these signals is friend, foe, or indifferent is not the topic of concern for this paper.

\section{Proposed Schemes}

In this section, brief introduction and flow diagram are provided for IPA, PS, and GA.

4.1. Interior Point Algorithm (IPA). Interior point methods (barrier methods) can be used for linear and nonlinear convex optimization problems. It uses either conjugate gradient step through a trust region or Newton step by using linear programming in order to get an optimum solution during each iteration [26]. The IPA has extensive applications and performs very well particularly in the presence of less local minima. However, its performance is superb even in the presence of more local minima when it is used as a local search optimizer with PSO or GA. For detailed applications and derivation of the algorithm, it is recommended that reader should see [27]. By observing such applications, in this work IPA is mainly used as a local search optimizer with GA.

4.2. Pattern Search (PS). Pattern search was introduced by Hookes and Jeeves in 1961 which is gradient or derivative free technique and can be used for both local and global optimization problems. Basically, PS works on mesh which is defined according to some specific rules. If no improvement is achieved in cost function at the mesh points of current iteration, then the mesh is polished and the process is repeated. It has applications in many fields, such as signal processing and soft computing [28]. In this work, PS is also mainly used as a local search optimizer with GA in which the best chromosome achieved through GA is given as the starting point to PS.

4.3. Genetic Algorithm (GA). GA is basically different from previously discussed algorithm (IPA and PS) and is applicable to a wide range of optimization problems. GA is more prominent and proficient algorithm than any other evolutionary computing technique due to its ease in conception and ease in implementation and more importantly less probable to get stuck in the presence of local optima. GA is being successfully applied to a wide range of applications from commerce to scientific research [29].

The steps for GA and GA-PS in the form of pseudocode are given below, while their flow diagram is shown in Figure 2.

Step 1 (initialization). In this step, we randomly generate $I$ number of chromosomes, where the length of each chromosome is $5 * P$. In each chromosome the first $P$ genes represent amplitudes, the second $P$ genes contain the frequencies, and the next $P$ genes represent the ranges, while the fourth and fifth $P$ genes represent elevation and azimuth angles, respectively, of the sources as follows:

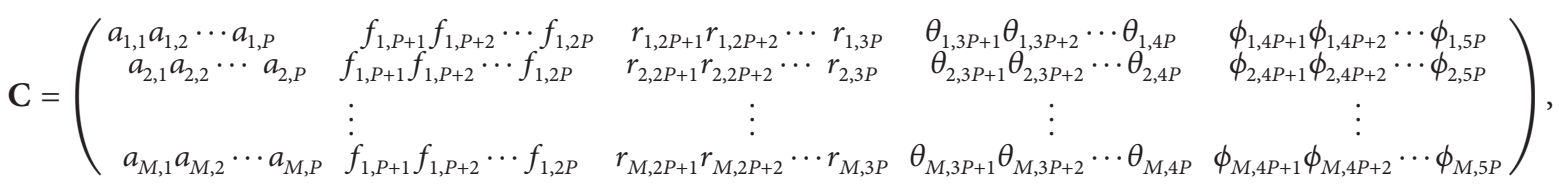

where

$$
\begin{gathered}
a_{i n} \in R: L_{l} \leq a_{i p} \leq H_{l}, \\
f_{i, P+p} \in R: f_{\min } \leq f_{i, P+p} \leq f_{\max }, \\
r_{i, 2 P+p} \in R: L_{r} \leq r_{i, 2 P+p} \leq H_{r},
\end{gathered}
$$

$$
\begin{gathered}
\theta_{i, 3 P+p} \in R: 0 \leq \theta_{i, 3 P+p} \leq \frac{\pi}{2}, \\
\phi_{i, 4 P+p} \in R: 0 \leq \phi_{i, 4 P+p} \leq 2 \pi ;
\end{gathered}
$$$$
\text { for } i=1,2, \ldots M, p=1,2, \ldots P \text {, }
$$ 
where $L_{l}$ and $l_{r}$ are the lowest, while $H_{l}$ and $H_{r}$ are the highest limits of signals amplitude and range, respectively.

Step 2 (fitness function). Our goal is to minimize the errors received for both subarrays. For $i$ th chromosome, it can be given as

$$
\operatorname{err}(i)=\operatorname{err}_{x}(i)+\operatorname{err}_{y}(i)
$$

$$
\begin{aligned}
& \operatorname{err}_{x}(i)=\frac{1}{2 Q_{x}} \sum_{m=-Q_{x}+1}^{Q_{x}}\left|w_{m, 0}-\widehat{w}_{m, 0}^{i}\right|^{2}+|| \mathbf{w}_{x N}^{H} \cdot \widehat{\mathbf{w}}_{x N}^{i}|-1|, \\
& \operatorname{err}_{y}(i)=\frac{1}{2 Q_{y}} \sum_{n=-Q_{y}+1}^{Q_{y}}\left|w_{0, n}-\widehat{w}_{0, n}^{i}\right|^{2}+|| \mathbf{w}_{y N}^{H} \cdot \widehat{\mathbf{w}}_{y N}^{i}|-1|
\end{aligned}
$$

where in (21), $w_{m, 0}$ and $w_{0, n}$ are defined in (8), respectively, while $\widehat{w}_{m, 0}^{i}$ and $\widehat{w}_{0, n}^{i}$ are given as

$$
\begin{aligned}
& \widehat{w}_{m, 0}^{i}=\sum_{p=1}^{P} \hat{c}_{p}^{i} e^{j\left(\left(\pi / f_{\max }\right)\left(\left(-m\left(\hat{c}_{P+p}^{i}\right) / 2\right) \sin \left(\hat{c}_{3 P+p}^{i}\right) \cos \left(\hat{c}_{4 P+p}^{i}\right)+\left(m^{2}\left(\hat{c}_{P+p}^{i}\right)^{2} / 16\left(\hat{c}_{2 P+p}^{i}\right)\right)\left(1-\sin ^{2}\left(\hat{c}_{3 P+p}^{i}\right) \cos ^{2}\left(\hat{c}_{4 P+p}^{i}\right)\right)\right)\right.}, \\
& \widehat{w}_{0, n}^{i}=\sum_{p=1}^{P} \widehat{c}_{p}^{i} e^{j\left(\left(\pi / f_{\max }\right)\left(\left(-n\left(\hat{c}_{P+p}^{i}\right) / 2\right) \sin \left(\widehat{c}_{3 P+p}^{i}\right) \sin \left(\hat{c}_{4 P+p}^{i}\right)+\left(n^{2}\left(\hat{c}_{P+p}^{i}\right)^{2} / 16\left(\hat{c}_{2 P+p}^{i}\right)\right)\left(1-\sin ^{2}\left(\widehat{c}_{3 P+p}^{i}\right) \sin ^{2}\left(\hat{c}_{4 P+p}^{i}\right)\right)\right)\right.} .
\end{aligned}
$$

Similarly, in (21), $\mathbf{w}_{x N}, \widehat{\mathbf{w}}_{x N}^{i}, \mathbf{w}_{y N}$, and $\widehat{\mathbf{w}}_{y N}^{i}$ can be defined as

$$
\begin{aligned}
& \mathbf{w}_{z N}=\frac{\mathbf{w}_{z}}{\left\|\mathbf{w}_{z}\right\|}, \\
& \widehat{\mathbf{w}}_{z N}^{i}=\frac{\widehat{\mathbf{w}}_{z}^{i}}{\left\|\widehat{\mathbf{w}}_{z}^{i}\right\|},
\end{aligned}
$$

where $z=x, y$.

Step 3 (termination criteria). The termination criteria depend on the following conditions if they are achieved:

(a) the objective function value is achieved which is predefined; that is, $\varepsilon \leq 10^{-7}$, or

(b) total number of iterations has been completed.

Step 4 (reproduction). New population is reproduced by using the operators of crossover, elitism, and mutation selection as shown in Table 1.

Step 5 (hybridization). In this important step, for further improvements, the best chromosome achieved through GA is given to PS and IPA as starting point. The parameter settings for IPA and PS are provided in Table 1.

Step 6 (storage). For better statistical analysis, store the global best of the current run and repeat Steps 2-5 for sufficient numbers of independent runs.

\section{Results and Discussion}

In this section, several simulations are performed to validate the proposed schemes. Initially, the comparison of proposed hybrid schemes is carried out with the individual performance of GA, IPA, and PS in terms of estimation accuracy, convergence rate, and proximity effects. At the end of this section, the comparison of proposed schemes is made with the traditional existing technique [15] using error as a figure of merit. We have used a MATLAB built-in optimization tool box, for which the parameter settings are provided in Table 1. All the values of frequencies, ranges, and DOA are taken in terms of Mega-Hertz $(\mathrm{MHz})$, wavelength $(\lambda)$, and radians (rad), respectively. Every time, we have used same number of sensors in both subarrays, where the reference sensor is common for both. The interelement spacing between the two consecutive sensors in each subarray is taken as $\lambda / 4$. Each result is averaged over 100 independent runs.

Case 1. In this case, the estimation accuracy of IPA, PS, GA, GA-IPA, and GA-PS is discussed for 2 sources. The CSCA consists of 9 sensors; that is, each subarray is composed of four sensors, while the reference sensor is common for them. In this case, no noise is added to the system. The desired values of amplitudes, frequencies, ranges, elevation, and azimuth angles are $a_{1}=6, f_{1}=30 \mathrm{MHz}, r_{1}=2 \lambda$, $\theta_{1}=0.2618 \mathrm{rad}$, and $\phi_{1}=2.0071 \mathrm{rad} ; a_{2}=4, f_{2}=60 \mathrm{MHz}$, $r_{2}=0.6 \lambda, \theta_{2}=1.1345 \mathrm{rad}$, and $\phi_{2}=2.9671 \mathrm{rad}$.

Although in this case, GA alone has produced fairly good estimation accuracy as provided in Table 2; however, it becomes even more accurate when hybridized with IPA and PS. Among all schemes, the GA-PS approach produced better results and maintained less error between desired values and estimated values. The second best scheme is GA-IPA, while GA alone provides the third best results.

Case 2. In this case, the estimation accuracy is discussed for 3 sources having values $a_{1}=3, f_{1}=40 \mathrm{MHz}, r_{1}=2.5 \lambda$, $\theta_{1}=0.4363 \mathrm{rad}$, and $\phi_{1}=1.0472 \mathrm{rad} ; a_{2}=1, f_{2}=70 \mathrm{MHz}$, $r_{2}=5 \lambda, \theta_{2}=0.7330 \mathrm{rad}$, and $\phi_{2}=2.1817 \mathrm{rad} ; a_{3}=7, f_{3}=$ $50 \mathrm{MHz}, r_{3}=0.2 \lambda, \theta_{3}=1.3963 \mathrm{rad}$, and $\phi_{3}=3.5779 \mathrm{rad}$. This time the array is composed of 13 sensors. Due to the increase of sources, the accuracy of IPA, PS, and GA has been significantly despoiled. However, as listed in Table 3, the accuracy of GA has improved when hybridized with IPA and PS. 
TABle 1: Parameter setting for GA, PS, and IPA.

\begin{tabular}{|c|c|c|c|c|c|}
\hline \multicolumn{2}{|c|}{ GA } & \multicolumn{2}{|c|}{ PS } & \multicolumn{2}{|c|}{ IPA } \\
\hline Parameters & Settings & Parameters & Setting & Parameters & Setting \\
\hline Population size & 240 & Starting point & $\begin{array}{c}\text { Best } \\
\text { chromosome } \\
\text { achieved by } \\
\text { GA }\end{array}$ & Starting point & $\begin{array}{c}\text { Best } \\
\text { chromosome } \\
\text { achieved by } \\
\text { GA }\end{array}$ \\
\hline $\begin{array}{l}\text { Number of } \\
\text { generations }\end{array}$ & 1000 & Polling order & Consecutive & $\begin{array}{c}\text { Subproblem } \\
\text { algorithm }\end{array}$ & $\begin{array}{c}\text { Idl } \\
\text { factorization }\end{array}$ \\
\hline $\begin{array}{l}\text { Migration } \\
\text { direction }\end{array}$ & Both ways & $\begin{array}{l}\text { Maximum } \\
\text { iteration }\end{array}$ & 1000 & $\begin{array}{c}\text { Maximum } \\
\text { perturbation }\end{array}$ & 0.1 \\
\hline Crossover fraction & 0.2 & $\begin{array}{c}\text { Function } \\
\text { evaluation }\end{array}$ & 17000 & $\begin{array}{c}\text { Minimum } \\
\text { perturbation }\end{array}$ & $1 e^{-8}$ \\
\hline Crossover & Heuristic & Mesh size & 01 & Scaling & $\begin{array}{c}\text { Objective and } \\
\text { constraint }\end{array}$ \\
\hline Function tolerance & $10-12$ & Expansion factor & 2.0 & Hessian & BFGS \\
\hline Initial range & $(0-1)$ & Contraction factor & 0.5 & Derivative type & $\begin{array}{l}\text { Central } \\
\text { difference }\end{array}$ \\
\hline Scaling function & Rank & Penalty factor & 100 & Penalty factor & 100 \\
\hline Selection & Stochastic uniform & Bind tolerance & $10-04$ & $\begin{array}{c}\text { Maximum function } \\
\text { evaluation }\end{array}$ & 50000 \\
\hline Elite count & 2 & Mesh tolerance & $10-07$ & Maximum iteration & 1000 \\
\hline Mutation function & Adaptive feasible & $\mathrm{X}$ tolerance & $10-06$ & $\mathrm{X}$ tolerance & $10-12$ \\
\hline
\end{tabular}

TABLE 2: Estimation accuracy of 2 sources using 9 sensors.

\begin{tabular}{lcccccccccc}
\hline Scheme & $a_{1}$ & $f_{1}(\mathrm{MHz})$ & $r_{1}(\lambda)$ & $\theta_{1}(\mathrm{rad})$ & $\phi_{1}(\mathrm{rad})$ & $a_{2}$ & $f_{2}(\mathrm{MHz})$ & $r_{2}(\lambda)$ & $\theta_{2}(\mathrm{rad})$ & $\phi_{2}(\mathrm{rad})$ \\
\hline Desired values & 6.0000 & 30.0000 & 2.0000 & 0.2618 & 2.0071 & 4.0000 & 60.0000 & 0.6000 & 1.1345 & 2.9671 \\
IPA & 6.0096 & 33.5342 & 2.0095 & 0.2714 & 2.0168 & 4.0095 & 63.5376 & 0.6095 & 1.1442 & 2.9767 \\
PS & 6.0050 & 32.7908 & 2.0051 & 0.2668 & 2.0123 & 4.0049 & 62.7546. & 0.6050 & 1.1396 & 2.9722 \\
GA & 6.0031 & 30.9765 & 2.0032 & 0.2649 & 2.0103 & 4.0032 & 60.9782 & 0.6030 & 1.1376 & 2.9703 \\
GA-IPA & 6.0020 & 30.2289 & 2.0019 & 0.2638 & 2.0091 & 4.0020 & 60.2415 & 0.6018 & 1.1367 & 2.9692 \\
GA-PS & 6.0007 & 30.1089 & 2.0008 & 0.2625 & 2.0078 & 4.0008 & 60.1063 & 0.6007 & 1.1352 & 2.9678 \\
\hline
\end{tabular}

TABLE 3: Estimation accuracy of 3 sources using 13 sensors.

\begin{tabular}{|c|c|c|c|c|c|c|c|c|c|c|c|c|c|c|c|}
\hline & $a_{1}$ & $\begin{array}{c}f_{1} \\
(\mathrm{MHz})\end{array}$ & $\begin{array}{l}r_{1} \\
(\lambda)\end{array}$ & $\begin{array}{c}\theta_{1} \\
(\mathrm{rad})\end{array}$ & $\begin{array}{c}\phi_{1} \\
(\mathrm{rad})\end{array}$ & $a_{2}$ & $\begin{array}{c}f_{2} \\
\mathrm{MHz})\end{array}$ & $\begin{array}{l}r_{2} \\
(\lambda)\end{array}$ & $\begin{array}{c}\theta_{2} \\
(\mathrm{rad})\end{array}$ & $\begin{array}{c}\phi_{2} \\
(\mathrm{rad})\end{array}$ & $a_{3}$ & $\begin{array}{c}f_{3} \\
(\mathrm{MHz})\end{array}$ & $\begin{array}{l}r_{3} \\
(\lambda)\end{array}$ & $\begin{array}{c}\theta_{3} \\
(\mathrm{rad})\end{array}$ & $\begin{array}{c}\phi_{3} \\
(\mathrm{rad})\end{array}$ \\
\hline & & 00 & & 0.4363 & 1.0472 & & 70.0000 & & & & & & & & \\
\hline 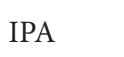 & & 46.98 & & .4920 & & & 64.3 & & 0.7888 & & & & & & 3.6337 \\
\hline & & & & & & & 75.8734 & & 0.7668 & & & & & & 3.6117 \\
\hline & & 43.7894 & & 0.4456 & 1.0565 & & 66.5682 & & 0.7423 & & & & & & 3.5871 \\
\hline & & 41.2187 & & & & 1.00 & 71.2654 & & 0.7396 & & & & & & 3.5847 \\
\hline & 3.0024 & 40.8903 & 2.5022 & 0.4388 & 1.0497 & 1.0024 & 70.8931 & 5.0025 & 0.7356 & 2.1841 & & 50.7969 & & 1.3986 & 3.5802 \\
\hline
\end{tabular}

The hybrid GA-PS technique proved to be the most accurate approach for three sources, while the second best approach is the other hybrid GA-IPA approach.

Case 3. In this case, the estimation accuracy of four near-field sources is discussed in the absence of noise where the CSCA is composed of 17 sensors. The desired values are $a_{1}=3.5, f_{1}=$
$65 \mathrm{MHz}, r_{1}=1 \lambda, \theta_{1}=0.4712 \mathrm{rad}$, and $\phi_{1}=0.1745 \mathrm{rad} ; a_{2}=$ $5, f_{2}=30 \mathrm{MHz}, r_{2}=6 \lambda, \theta_{2}=0.8727 \mathrm{rad}$, and $\phi_{2}=2.0420$ rad; $a_{3}=2, f_{3}=85 \mathrm{MHz}, r_{3}=10 \lambda, \theta_{3}=1.2741 \mathrm{rad}$, and $\phi_{3}=2.7925 \mathrm{rad} ; a_{4}=8, f_{4}=25 \mathrm{MHz}, r_{4}=4 \lambda, \theta_{4}=1.5184$ $\mathrm{rad}$, and $\phi_{4}=4.4506 \mathrm{rad}$. One can see from Table 4 that the estimation accuracy of all schemes degraded as we have faced more local minima in this case. However, even in this case, the 


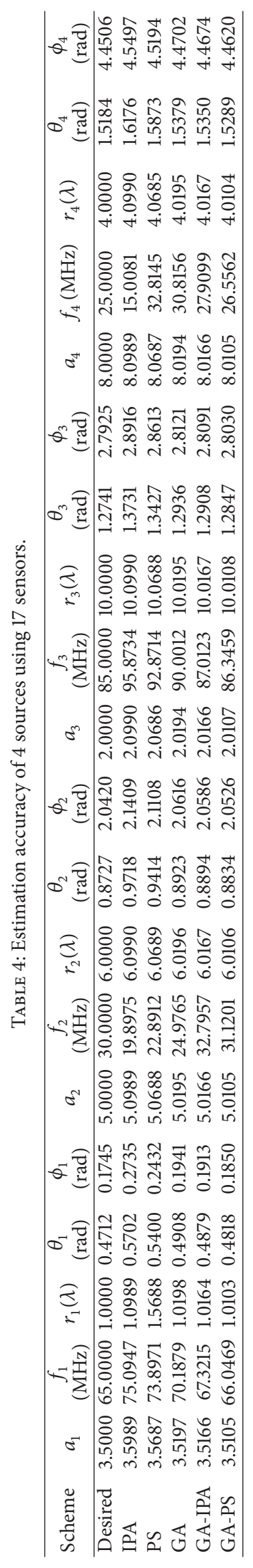


TABLE 5: Estimation accuracy for 3 sources at $\mathrm{SNR}=5 \mathrm{~dB}$.

\begin{tabular}{lccccccccccccccc}
\hline Scheme & $a_{1}$ & $\begin{array}{c}f_{1} \\
(\mathrm{MHz})\end{array}$ & $\begin{array}{c}r_{1} \\
(\lambda)\end{array}$ & $\begin{array}{c}\theta_{1} \\
(\mathrm{rad})\end{array}$ & $\begin{array}{c}\phi_{1} \\
(\mathrm{rad})\end{array}$ & $a_{2}$ & $\begin{array}{c}f_{2} \\
(\mathrm{MHz})\end{array}$ & $\begin{array}{c}r_{2} \\
(\lambda)\end{array}$ & $\begin{array}{c}\theta_{2} \\
(\mathrm{rad})\end{array}$ & $\begin{array}{c}\phi_{2} \\
(\mathrm{rad})\end{array}$ & $\begin{array}{c}a_{3} \\
\begin{array}{l}f_{3} \\
(\mathrm{MHz})\end{array}\end{array} \begin{array}{c}r_{3} \\
(\lambda)\end{array} \begin{array}{c}\theta_{3} \\
(\mathrm{rad})\end{array}$ & $\begin{array}{c}\phi_{3} \\
(\mathrm{rad})\end{array}$ \\
\hline Desired & 3.0000 & 70.0000 & 6.0000 & 0.2618 & 0.6109 & 1.0000 & 45.0000 & 2.4000 & 0.7854 & 2.4435 & 7.0000 & 30.0000 & 4.3000 & 1.4835 & 3.7525 \\
IPA & 3.3711 & 78.8790 & 6.1712 & 0.4329 & 0.7820 & 1.4711 & 54.9876 & 2.5712 & 0.9567 & 2.6145 & 7.1710 & 39.8791 & 4.4711 & 1.6547 & 3.9235 \\
PS & 3.2047 & 77.2137 & 6.1047 & 0.3665 & 0.7156 & 1.3047 & 53.1124 & 2.1047 & 0.8901 & 2.5483 & 7.1045 & 38.1236 & 4.4048 & 1.5884 & 3.8572 \\
GA & 3.1824 & 75.8711 & 6.0423 & 0.3142 & 0.6534 & 1.2422 & 49.8879 & 2.4425 & 0.8279 & 2.4860 & 7.0426 & 35.4398 & 4.3424 & 1.5259 & 3.7950 \\
GA-IPA & 3.0584 & 72.3298 & 6.0385 & 0.3002 & 0.6494 & 1.1385 & 47.6675 & 2.4384 & 0.8239 & 2.4820 & 7.0382 & 32.9983 & 4.3384 & 1.5220 & 3.7910 \\
GA-PS & 3.0357 & 71.1903 & 6.0156 & 0.2775 & 0.6266 & 1.0958 & 46.1290 & 2.4158 & 0.8013 & 2.4592 & 7.0158 & 31.6722 & 4.3158 & 1.4993 & 3.7684 \\
\hline
\end{tabular}

TABLE 6: Proximity effect of DOA of three sources and 17 sensors at $\mathrm{SNR}=10 \mathrm{~dB}$.

\begin{tabular}{lccccccc}
\hline Scheme & $\theta_{1}(\mathrm{rad})$ & $\phi_{1}(\mathrm{rad})$ & $\theta_{2}(\mathrm{rad})$ & $\phi_{2}(\mathrm{rad})$ & $\theta_{3}(\mathrm{rad})$ & $\phi_{3}(\mathrm{rad})$ & $\%$ convergence \\
\hline Desired values & 0.6981 & 1.9199 & 0.7679 & 1.9897 & 0.8378 & 2.0595 & - \\
IPA & 0.8203 & 2.0402 & 0.8901 & 2.1118 & 0.9599 & 2.1817 & 1 \\
PS & 0.7941 & 2.0351 & 0.8849 & 2.1049 & 0.9512 & 2.1712 & 2.1031 \\
GA & 0.7400 & 1.9600 & 0.8116 & 2.0298 & 0.8796 & 2.0857 & 7 \\
GA-IPA & 0.7208 & 1.9408 & 0.7906 & 2.0141 & 0.8587 & 2.0717 & 80 \\
GA-PS & 0.7103 & 1.9303 & 0.7821 & 2.0019 & 0.8482 & 2 \\
\hline
\end{tabular}

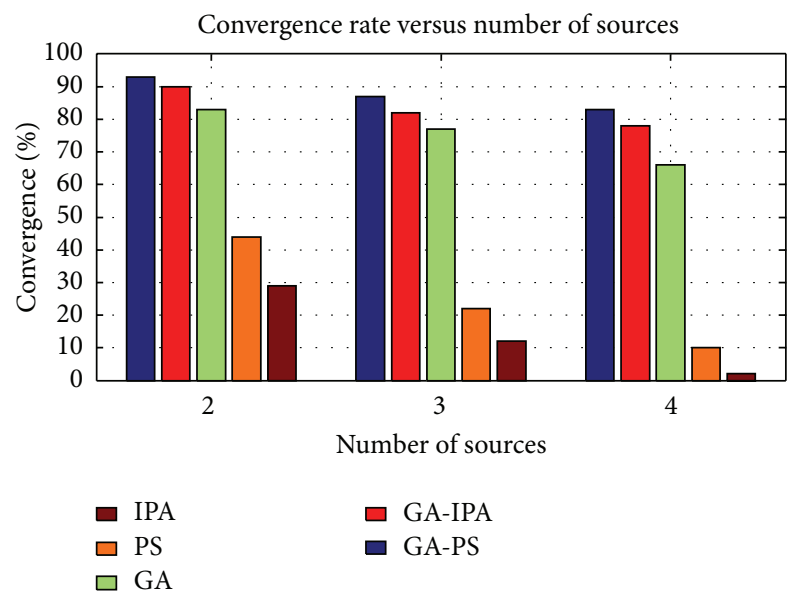

Figure 3: Convergence rate versus number of sources.

hybrid approaches especially the GA-PS performed well and made a close estimate of desired response. The second best scheme is again the other hybrid GA-IPA approach.

Case 4. In Figure 3, convergence rate is shown for each scheme against different number of sources. From convergence, we mean, the total number of times a particular technique achieved its goal. In this case, we have taken the same two sources as given in Case 1, but this time the CSCA consists of 17 sensors for each number of sources. The bar graph shows that the hybrid GA-PS technique has converged many number of times as compared to the remaining approaches for all sources. The second best convergence rate

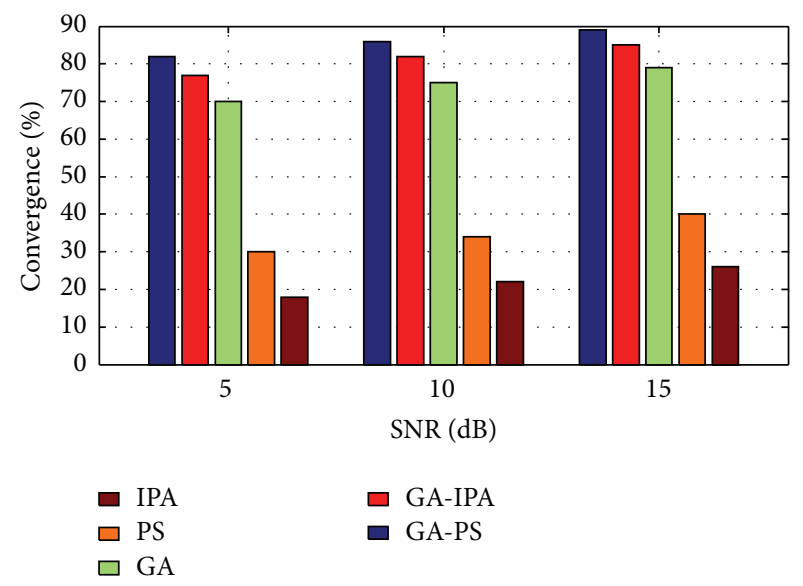

FIGURE 4: Convergence rate versus SNR.

is maintained by GA-IPA, while the third best scheme is GA alone.

Case 5. In this case, the estimation accuracy is checked in the presence of low signal to noise ratio (SNR). The value of SNR is $5 \mathrm{~dB}$, while the array has 13 sensors. The desired values of amplitude, frequency, ranges, elevation and azimuth angles of 3 sources are $a_{1}=3, f_{1}=70 \mathrm{MHz}, r_{1}=6 \lambda$, $\theta_{1}=0.2618 \mathrm{rad}$, and $\phi_{1}=0.6109 \mathrm{rad} ; a_{2}=1, f_{2}=45 \mathrm{MHz}$, $r_{2}=2.4 \lambda, \theta_{2}=0.7854 \mathrm{rad}$, and $\phi_{2}=2.4435 \mathrm{rad} ; a_{3}=7, f_{3}=$ $30 \mathrm{MHz}, r_{3}=4.3 \lambda, \theta_{3}=1.4835 \mathrm{rad}$, and $\phi_{3}=3.7525 \mathrm{rad}$. As provided in Table 5, due to low SNR' the accuracy of all schemes is despoiled. However, the hybrid GA-PS scheme is robust enough to produce better results even in the presence 


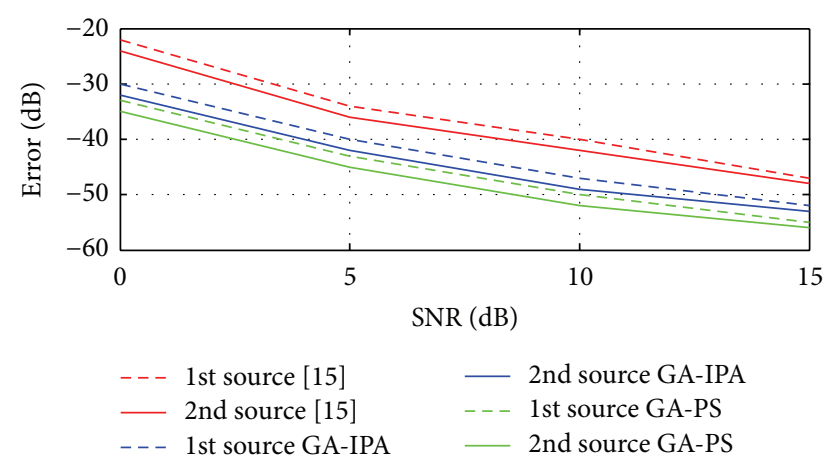

FIGURE 5: Error estimation of the frequencies versus SNR.

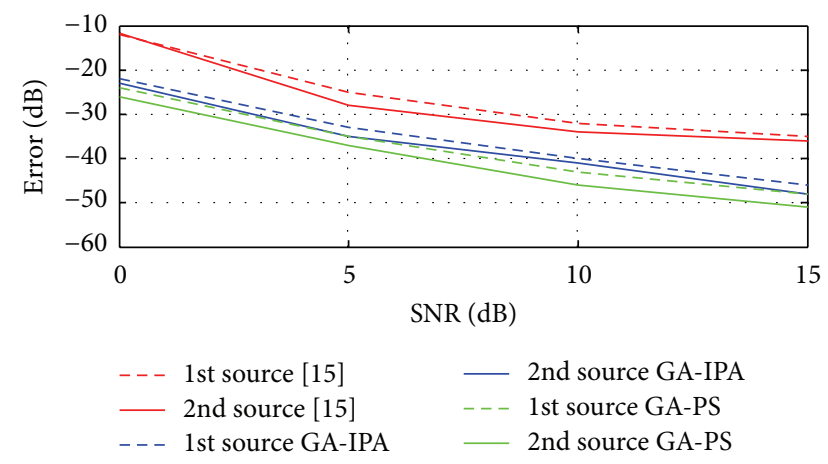

FIGURE 6: Error estimation of the azimuth angles versus SNR.

of low SNR. The second best result is produced by the other hybrid GA-IPA scheme.

Case 6. In Figure 4, the convergence rate of each scheme is evaluated against noise and it has been shown that the convergence rate of all schemes degraded at low values of SNR. However, with the increase of SNR, the convergence rate of each scheme has improved. Again, the hybrid GAPS has shown fairly good robustness against all the values of SNR.

Case 7. In this case, the proximity effect of DOA of three sources is evaluated in terms of estimation accuracy and convergence rate in the presence of $10 \mathrm{~dB}$ noise. As given in Table 6, due to proximity and low SNR, we have faced more local minima. However, once again one can see that the hybrid GA-PS produced fairly good results in terms of accuracy and convergence rate even in this case, while the second best result is given by GA-IPA.

Case 8. In this case, we have compared the proposed two hybrid schemes with traditional technique given in [15]. Basically, in [15], Liang et al. have proposed a cumulants based technique to estimate the 4D parameters (Frequency, range, elevation angle, and azimuth angle) of near-field sources. In [15], mean square error (MSE) is used, while in the current work, the error is the combination of MSE and correlation between the desired and estimated vectors as discussed in

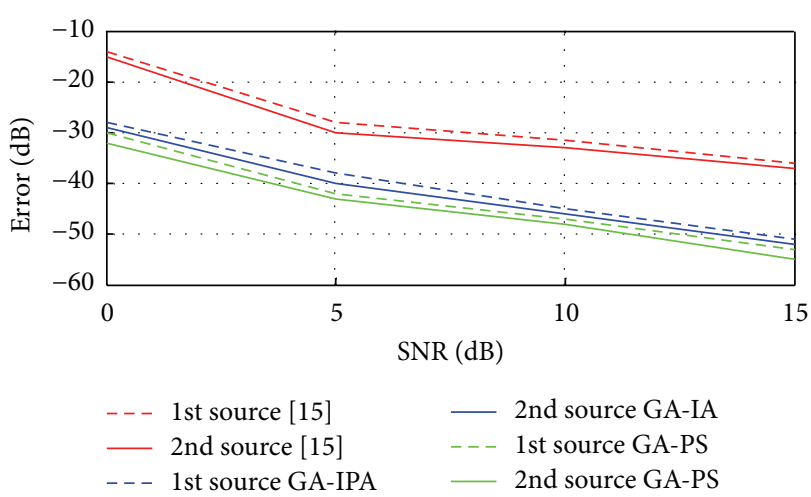

FIGURE 7: Error estimation of the elevation angles versus SNR.

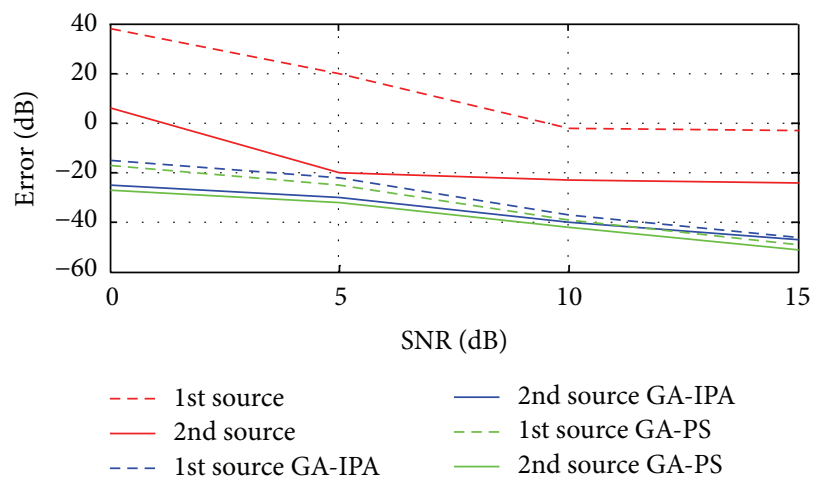

FIGURE 8: Error estimation of the ranges versus SNR.

Section 3. For these simulations, two sources are considered in the presence of noise. The values of the two sources are exactly the same as given above in Case 1. Figures 5, 6,7 , and 8 have shown the error for frequency, azimuth angle, elevation angle, and range of two near-field sources by using [15] and the two proposed hybrid schemes. One can clearly observe that, in each case (especially for range estimation), the proposed schemes have maintained fairly minimum error as compared to [15]. Besides, [15] is unable to estimate the amplitude, while our proposed schemes have shown satisfactory error for amplitude estimation as shown in Figure 9.

\section{Conclusion and Future Work}

In this work, we have mainly developed two hybrid schemes (GA-IPA and GA-PS) to estimate the 5D parameters (amplitude, frequency, range, elevation angle, and azimuth angle) of sources located in the near field of the sensors array. A new multiobjective fitness function was developed, which is the combination of MSE and correlation between normalized desired and normalized estimated vectors. It requires only single snapshot. The two hybrid schemes have shown good performance as compared to their individual responses in terms of estimation accuracy, convergence rate, and so forth. The proposed schemes have also shown good results as compared to traditional technique by using an error as a 


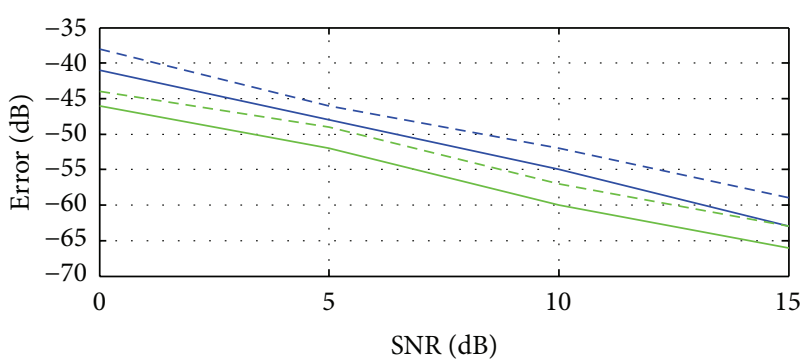

$\begin{array}{llll}--- & 1 \text { st } \text { source GA-IPA } & -- & \text { 1st source GA-PS } \\ - & \text { 2nd source GA-IPA } \quad-\quad \text { 2nd source GA-PS }\end{array}$

FIGURE 9: Error estimation of the amplitudes versus SNR.

figure of merit. However, the hybrid GA-PS proved to be the best approach among them for the joint estimation of amplitude, frequency, range, elevation angle, and azimuth angle of near-field sources.

In future, one can check the same approach for null steering and beam steering in the field of adaptive beamforming.

\section{Conflict of Interests}

The authors declare that there is no conflict of interests regarding the publication of this paper.

\section{References}

[1] H. Krim and M. Viberg, "Two decades of array signal processing research: the parametric approach," IEEE Signal Processing Magazine, vol. 13, no. 4, pp. 67-94, 1996.

[2] R. O. Schmidt, "Multiple emitter location and signal parameter estimation," IEEE Transactions on Antennas and Propagation, vol. 34, no. 3, pp. 276-280, 1986.

[3] S. M. Kay, Fundamentals of Statistical Signal Processing: Estimation Theory, vol. 1 of Prentice Hall Signal Processing Series, Prentice-Hall, Upper Saddle River, NJ, USA, 1st edition, 1993.

[4] T. K. Sarkar and O. Pereira, "Using the matrix pencil method to estimate the parameters of a sum of complex exponentials," IEEE Antennas and Propagation Magazine, vol. 37, no. 1, pp. 4855, 1995.

[5] R. Roy and T. Kailath, "ESPRIT-estimation of signal parameters via rotational invariance techniques," IEEE Transactions on Acoustics, Speech, and Signal Processing, vol. 37, no. 7, pp. 984995, 1989.

[6] A. Swindlehurst and T. Kailath, "Passive direction-of-arrival and range estimation for near-field sources," in Proceedings of the 4th Annual ASSP Workshop on Spectrum Estimation and Modeling, pp. 123-128, Minneapolis, Minn, USA, August 1988.

[7] E. Grosicki, K. Abed-Meraim, and Y. Hua, "A weighted linear prediction method for near-field source localization," IEEE Transactions on Signal Processing, vol. 53, no. 10, part 1, pp. 36513660, 2005.

[8] D. Starer and A. Nehorai, "Passive localization on near-field sources by path following," IEEE Transactions on Signal Processing, vol. 42, no. 3, pp. 677-680, 1994.

[9] J.-F. Chen, X.-L. Zhu, and X.-D. Zhang, "A new algorithm for joint range-DOA-frequency estimation of near-field sources,"
EURASIP Journal on Applied Signal Processing, vol. 2004, Article ID 105173, pp. 386-392, 2004.

[10] Y. Wu, L. Ma, C. Hou, G. Zhang, and J. Li, "Subspace-based method for joint range and DOA estimation of multiple nearfield sources," Signal Processing, vol. 86, no. 8, pp. 2129-2133, 2006.

[11] N. Kabaoğlu, H. A. Çırpan, E. Çekli, and S. Paker, "Deterministic maximum likelihood approach for 3-D near field source localization," AEU-International Journal of Electronics and Communications, vol. 57, no. 5, pp. 345-350, 2003.

[12] R. N. Challa and S. Shamsunder, "Passive near-field localization of multiple non-Gaussian sources in 3-D using cumulants," Signal Processing, vol. 65, no. 1, pp. 39-53, 1998.

[13] K. Abed-Meraim and Y. Hua, "3-D near field source localization using second order statistics," in Proceedings of the 31st Asilomar Conference on Signals, Systems \& Computers, vol. 2, pp. 13071311, Pacific Grove, Calif, USA, November 1997.

[14] D.-S. Yang, J. Shi, and B.-S. Liu, "A new near-field source localization algorithm based on generalized esprit," in Proceedings of the 4th IEEE Conference on Industrial Electronics and Applications (ICIEA '09), pp. 1115-1120, Xi'an, China, May 2009.

[15] J. Liang, S. Yang, J. Zhang, L. Gao, and F. Zhao, “4D nearfield sourcelocalization using cumulant," EURASIP Journal on Advances in Signal Processing, vol. 2007, Article ID 17820, 10 pages, 2007.

[16] J. H. Holland, Adaptation in Natural and Artificial Systems: An Introductory Analysis with Applications to Biology, Control, and Artificial Intelligence, Complex Adaptive Systems, The MIT Press, Cambridge, Mass, USA, 1975.

[17] J. Kennedy and R. Eberhart, "Particle swarm optimization," in Proceedings of the IEEE International Conference on Neural Networks (ICNN '96), vol. 4, pp. 1942-1948, Perth, Wash, USA, December 1995.

[18] R. Storn and K. Price, "Differential evolution-a simple and efficient heuristic for global optimization over continuous spaces," Journal of Global Optimization, vol. 11, no. 4, pp. 341359, 1997.

[19] Z. Fawad, I. M. Qureshi, J. A. khan, and Z. U. khan, "An application of artificial intelligence for the joint estimation of amplitude and two-dimensional direction of arrival of far field sources using 2-L-shape array," International Journal of Antennas and Propagation, vol. 2013, Article ID 593247, 10 pages, 2013.

[20] M. Atique-ur-Rehman, F. Zaman, I. M. Qureshi, and Y. A. Shiekh, "Null and sidelobes adjustment of damaged array using hybrid computing," in Proceedings of the IEEE International Conference on Emerging Technologies (ICET '12), pp. 483-484, Islamabad, Pakistan, October 2012.

[21] F. Zaman, I. Qureshi, A. Malik, and U. Khan, "Real time direction of arrival estimation in noisy environment using particle swarm optimization with single snapshot," Research Journal of Applied Sciences, Engineering \& Technology, vol. 4, no. 13, pp. 1949-1952, 2012.

[22] F. Zaman, J. A. Khan, Z. U. Khan, and I. M. Qureshi, “An application of hybrid computing to estimate jointly the amplitude and direction of arrival with single snapshot," in Proceedings of the 10th IEEE International Bhurban Conference on Applied Sciences and Technology (IBCAST '13), pp. 15-19, Islamabad, Pakistan, January 2013.

[23] F. Zaman, I. M. Qureshi, A. Naveed, J. A. Khan, and R. M. Asif Zahoor, "Amplitude and directional of arrival estimation: 
comparison between different techniques," Progress in Electromagnetics Research B, vol. 39, pp. 319-335, 2012.

[24] F. Zaman, I. M. Qureshi, A. Naveed, and Z. U. Khan, "Joint estimation of amplitude, direction of arrival and rang of near field sources using memetic computing," Progress in Electromagnetics Research C, vol. 31, pp. 199-213, 2012.

[25] T. W. Anderson, "Asymtotic theory of principal component analysis," Annals of Mathematical Statistics, vol. 34, no. 1, pp. 122-148, 1963.

[26] R. A. Waltz, J. L. Morales, J. Nocedal, and D. Orban, “An interior algorithm for nonlinear optimization that combines line search and trust region steps," Mathematical Programming, vol. 107, no. 3, pp. 391-408, 2006.

[27] S. J. Wright, Primal-Dual Interior-Point Methods, SIAM, Philadelphia, Pa, USA, 1997.

[28] V. Torczon, "On the convergence of pattern search algorithms," SIAM Journal on Optimization, vol. 7, no. 1, pp. 1-25, 1997.

[29] B. Addad, S. Amari, and J.-J. Lesage, "Genetic algorithms for delays evaluation in networked automation systems," Engineering Applications of Artificial Intelligence, vol. 24, no. 3, pp. 485490, 2011. 

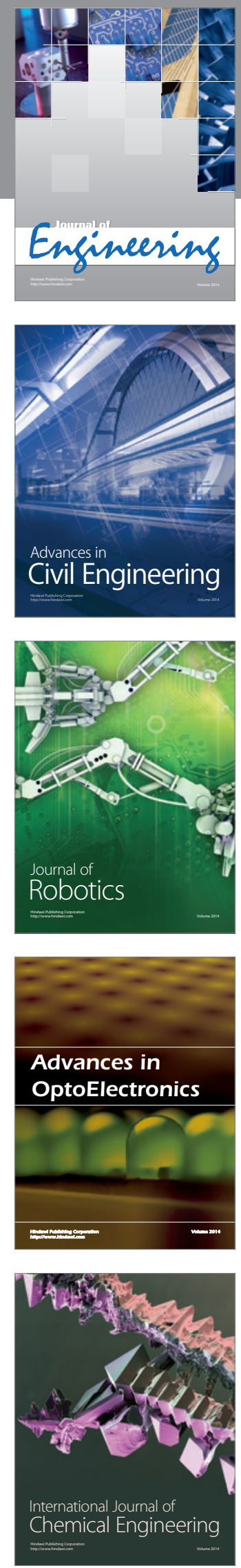

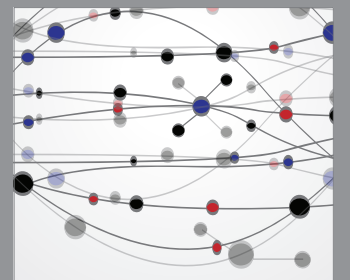

The Scientific World Journal
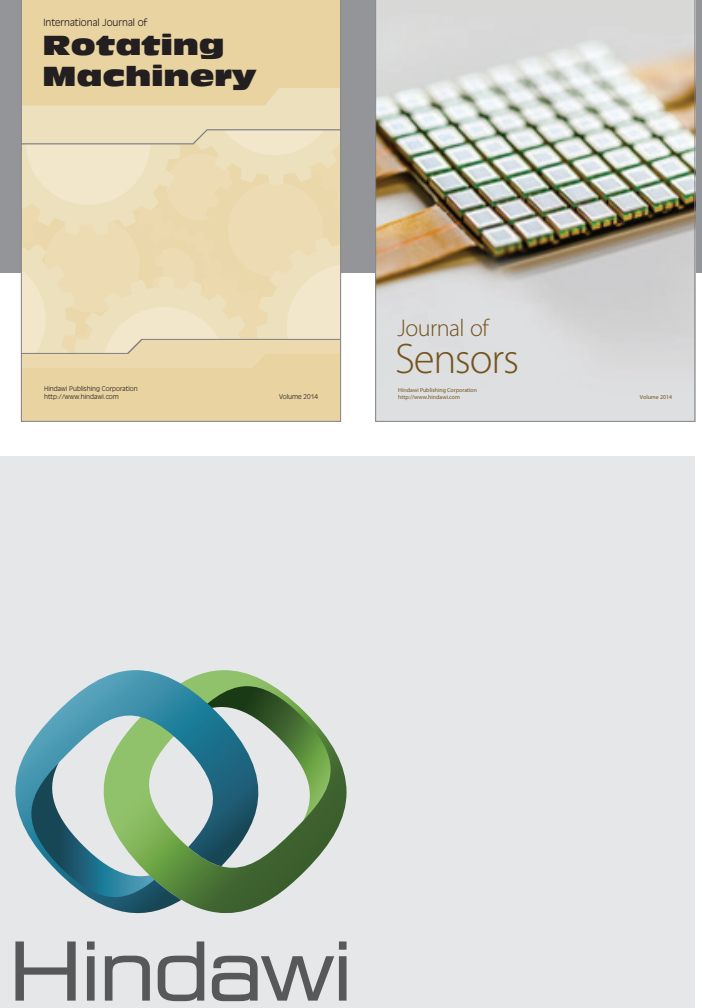

Submit your manuscripts at http://www.hindawi.com
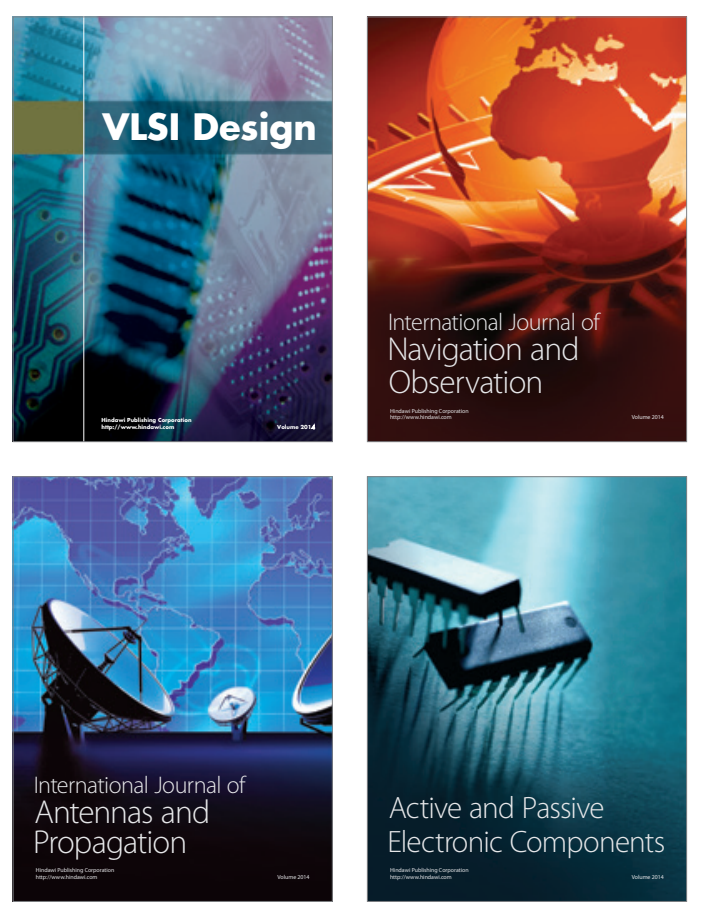
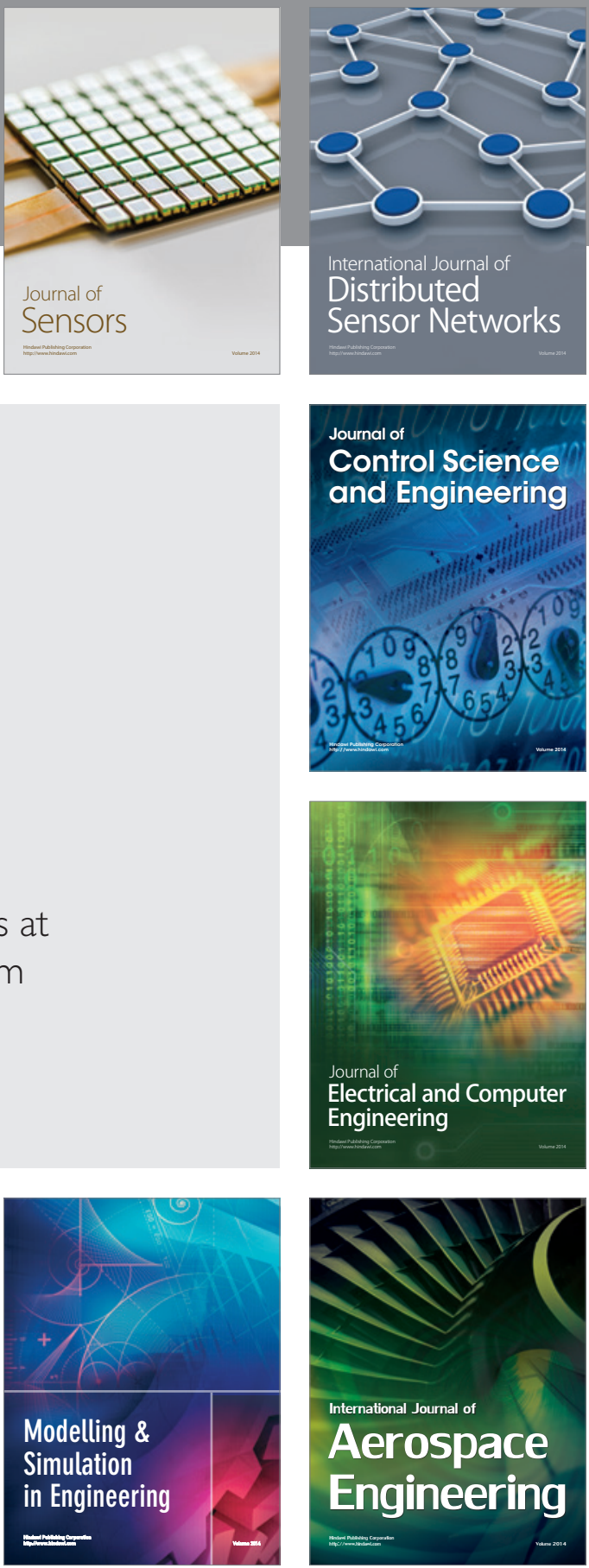

Journal of

Control Science

and Engineering
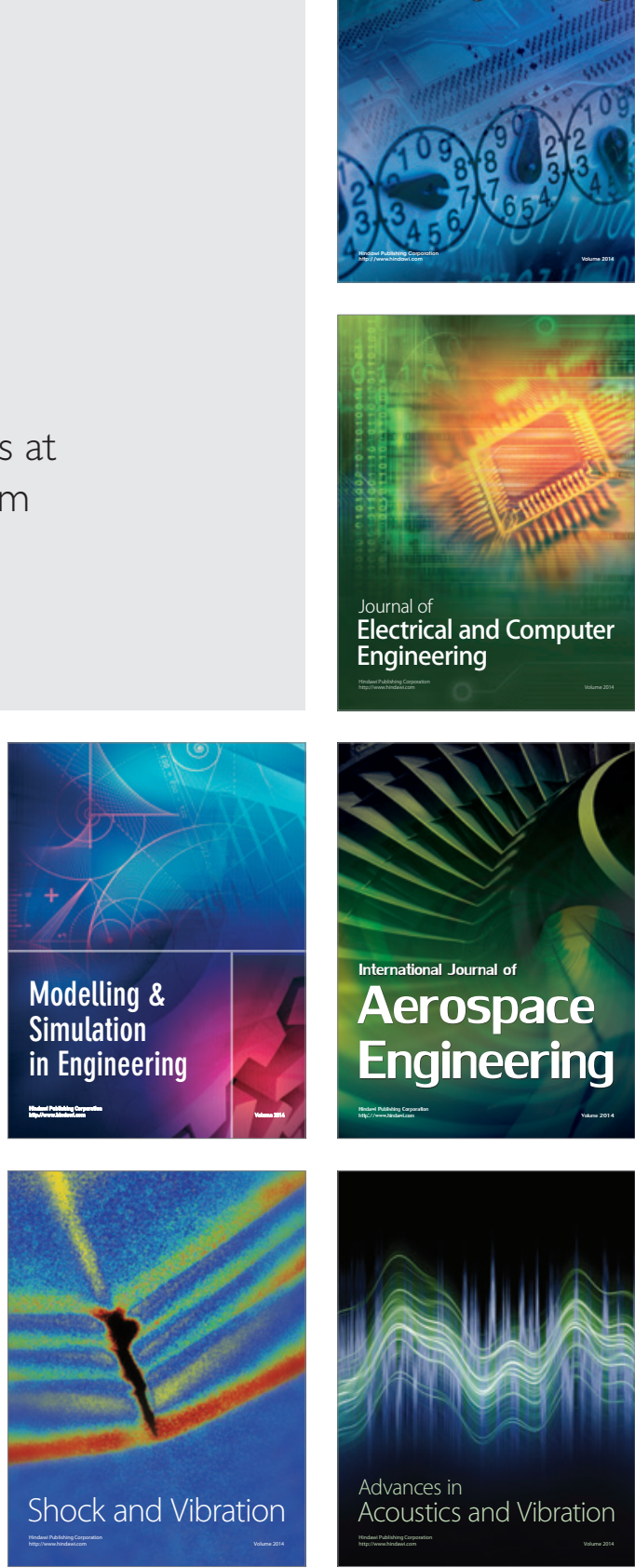